
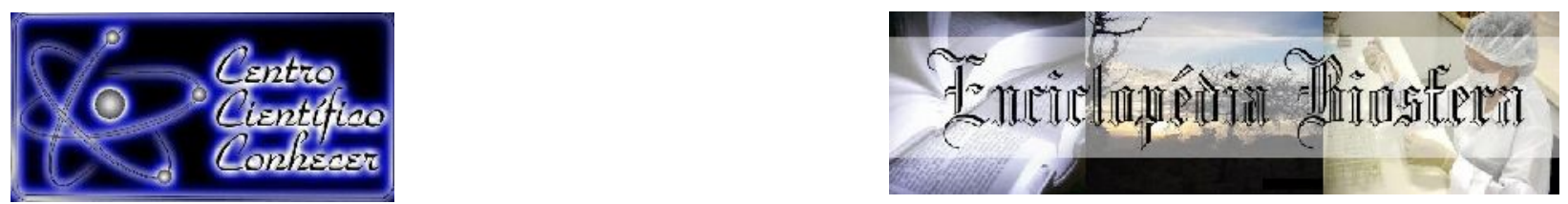

\title{
DESENVOLVIMENTO DE CÓDIGOS COMPUTACIONAIS, EM LINGUAGEM SCILAB, PARA A DETERMINAÇÃO DE PARÂMETROS CINÉTICOS DE REAÇÕES QUÍMICAS NÃO REVERSÍVEIS
}

\author{
Bruna Sgarlate ${ }^{1}$, Priscila Pereira Silva ${ }^{2}$, Nádia Guimarães Sousa $^{2}$
}

${ }^{1}$ Bacharel em Engenharia Química da Universidade Federal do Triângulo Mineiro, Uberaba, Brasil.

${ }^{2}$ Professora Doutora da Universidade Federal do Triângulo Mineiro, Uberaba, Brasil. E-mail: nadia.sousa@uftm.edu.br

Recebido em: 04/10/2019 - Aprovado em: 30/11/2019 - Publicado em: 15/12/2019 DOI: 10.18677/EnciBio_2019B20

\section{RESUMO}

Com o desenvolvimento tecnológico, a utilização de softwares para simular sistemas tornou-se cada vez mais comum dentro da Engenharia. Muitas vezes o estudo e resolução de problemas em qualquer área das engenharias não é um trabalho muito simples uma vez que a modelagem e caracterização de processos torna-se uma tarefa complexa de acordo com o nível de detalhamento requerido. Dessa forma, o objetivo deste trabalho é o desenvolvimento de códigos computacionais a partir do software livre Scilab para a determinação dos parâmetros cinéticos de reações químicas não reversíveis. Os códigos fornecem os resultados de acordo com o método de determinação de parâmetros cinéticos escolhido pelo usuário, dentre eles estão o Método Integral, Método Diferencial, Método dos Pontos Igualmente Espaçados, Método das Taxas Iniciais e Método dos Mínimos Quadrados. Os códigos apresentam uma interface interativa com o usuário, não necessitando assim, nenhum tipo de programação prévia. Os dados devem sem inseridos de acordo com a necessidade requerida de cada método e fica a critério do usuário a unidade a ser arbitrada. Os códigos podem ser usados como ferramenta de estudo da disciplina e tem como finalidade despertar o senso crítico dos usuários perante a uma variação sistêmica de um processo, e eles podem ser obtidos realizando o download através do site criado para este fim, disponível em:< https://crsolve.wixsite.com/solve>.

PALAVRAS-CHAVE: Cinética química, parâmetros cinéticos, simulação.

\section{THE COMPUTACIONAL CODE DEVELOPMENT, USING SCILAB LANGUAGE, IN THE DETERMINATION OF KINETIC PARAMETERS OF NON REVERSIBLE CHEMICAL REACTIONS}

\begin{abstract}
With the advancement of technology, the use of software to simulate systems has become more and more common inside the engineering. Many times, the study and problem solving of any field of engineering isn't an easy job to be done, since process modelling and characterization is a complex task depending on the level of detail required. Hence, the objective of this paper is the computational aided code
\end{abstract}


development, using the Scilab free software, in the determination of kinetic parameters of non reversible chemical reactions. The codes provide the results according to the method of determination of the kinetic parameters chosen by the user, among them the Integral Method, Differential Method, Equally Spaced Points Method, Initial Rates Method and Least Squares Method. The codes have an interactive interface with the user, sparing any kind of previous programming. Data must be inserted according to the required needs of the method, and the user can choose the best units for his or her purpose. The codes can be used as a study tool for this subject, and aims to awake the users' critical thinking towards a systematic process deviation, and they can be downloaded from a website created to this intent, available in :< https://crsolve.wixsite.com/solve>.

KEYWORDS: Chemical kinetics, kinetics parameters, simulation.

\section{INTRODUÇÃO}

Com a globalização e as constantes inovações tecnológicas, o sistema industrial passou por alterações significativas, as quais foram as principais responsáveis pelas mudanças e desenvolvimento de uma sociedade, que atualmente acompanha uma transformação tecnológica sem precedentes e precisa adaptar-se a essa realidade (SAKURAI; ZUCHI, 2018).

Devido ao ambiente industrial altamente competitivo que exige a modernização e otimização dos processos de fabricação tem-se o surgimento da indústria 4.0, que visa mudar o cenário industrial por meio do desenvolvimento e novas tecnologias (MAYNARD, 2015).

Um dos elementos de maior relevância para o desenvolvimento e otimização de processos é a simulação computacional, no qual são criados modelos que representam os sistemas reais (ROSEN et al., 2015). Esses modelos fornecem a possibilidade de avaliações sobre um modelo matemático fiel à realidade, em contraste com a realização de testes experimentais na planta. Tal técnica pode aumentar a produtividade, sem que o produto perca a qualidade, além de possibilitar a redução de custos (RÜßMANN et al., 2015).

A representação dos processos através de equações matemáticas é conhecida como modelagem de processo. Quanto maior a aproximação da realidade, mais complexos serão os modelos matemáticos encontrados (SCHULTZ et al., 2014).

As habilidades adquiridas através das disciplinas de crivo tecnológico como termodinâmica, fenômenos de transferência de calor e massa, mecânica dos fluidos, operações unitárias, processos industriais, automação e controle, são frequentes na formação de um engenheiro. Todavia, apenas o profissional do ramo da engenharia química agrega conhecimentos sobre cinéticas e cálculo de reatores que oferecem ferramentas e fundamentos básicos para a resolução de problemas característicos e desenvolvimento de projetos (ANGRISANO, 2016).

O estudo da cinética química visa compreender as diversas relações entre as velocidades das reações químicas e os fatores que as influenciam, tais como temperatura, concentração de reagente, presença ou ausência de catalisadores, entre outros (FERREIRA et al., 2014). A influência que a temperatura traz é que, quanto maior a temperatura em que reação química ocorre, maior será a energia cinética das moléculas envolvidas, o que promove uma agitação mais intensa nas partículas, e assim a frequência de colisão entre elas aumenta (SOUZA et. al., 2016). A cinética química visa o desenvolvimento de um mecanismo plausível para a 
reação ou a obtenção de uma equação ou um sistema de equações diferenciais e algébricas associadas entre si para definir as leis de velocidade de todas as etapas da reação (SILVEIRA, 2015).

Este trabalho tem o intuito de ressaltar os benefícios que a modelagem e simulação desempenham na área e como o uso de softwares, nesse caso específico o software livre Scilab, auxiliam no estudo de certas áreas da engenharia de modo a facilitar seu entendimento. Logo, o objetivo geral desse trabalho é o desenvolvimento de códigos, utilizando o software livre Scilab, para a determinação de parâmetros cinéticos de reações não reversíveis.

\section{MATERIAIS E MÉTODOS}

O Scilab é um software científico livre e gratuito para computação numérica que fornece um poderoso ambiente computacional aberto para aplicações científicas e de engenharia. Apresenta uma grande versatilidade devido a uma série de funcionalidades e capacidade de intercomunicação com outros softwares (SCILAB, 2019). Este trabalho possui natureza teórico-computacional e utilizou apenas o software livre, Scilab, para realizar as rotinas computacionais.

\section{Determinação de Parâmetros Cinéticos}

Os parâmetros cinéticos da taxa de reação são a constante cinética $(k)$ e as ordens de reação $(n$ e $m$ ) em relação a cada componente. $O$ efeito da temperatura está na velocidade específica e para determiná-las precisam ser determinados o fator de frequência $\left(k_{0}\right)$ e a energia de ativação $\left(E_{a}\right)$ (SCHMAL, 2013).

Segundo Fogler (2013) a coleta de dados cinéticos para reações homogêneas ocorre, frequentemente, em reatores batelada. Essa determinação é geralmente obtida através da medição da concentração de um componente em função do tempo. Se a variável medida não for a concentração deve-se reescrever o balanço molar em termos dessa variável. Dada uma reação irreversível de ordem $\mathrm{n}$ do tipo $A \rightarrow B$, tem-se que a equação genérica da taxa é dada pela equação (1).

$$
-\frac{d C_{A}}{d t}=-r_{A}=k C_{A}^{n}
$$

em que $k$ é a constante cinética da velocidade, $\mathrm{C}_{\mathrm{A}}$ é a concentração molar do reagente $A$ em um determinado tempo de reação, e $n$ é a ordem da reação em relação ao reagente $A$ (FOGLER, 2013).

Segundo Pereira (2019), a ordem de uma reação química pode ser definida como a soma das potências dos termos de concentração que estão presentes na equação de velocidade, e que pode ser obtida a partir de dados experimentais, em grande parte das vezes, sem o conhecimento prévio do real mecanismo da reação. Dessa forma, os parâmetros cinéticos, ordem e constante de velocidade, podem ser determinados a partir de métodos de tratamento de dados descritos a seguir.

\section{Método Diferencial}

O método diferencial pode ser ilustrado considerando uma reação acontecendo em um reator batelada a volume constante em que é registrada a variação da concentração com o tempo. A partir da linearização da equação (1), pode ser escrita a equação (2) (PEREIRA, 2019). 


$$
\ln \left(-\frac{d C_{A}}{d t}\right)=\ln (k)+n \ln \left(C_{A}\right)
$$

Logo, a ordem da reação $(n)$ é o coeficiente angular da reta obtida através da equação (2) e a constante cinética ( $k$ ) pode ser calculada através da exponencial do seu coeficiente linear.

\section{Método dos Pontos Igualmente Espaçados}

As fórmulas de diferenciação, abordadas nesse método, podem ser utilizadas quando os pontos experimentais relativos à variável independente estão igualmente espaçados, tais como $t_{1}-t_{0}=t_{2}-t_{1} \equiv \Delta t$. As fórmulas de diferenciação de três pontos são representadas pelas equações (3) - (5).

Primeiro ponto:

$$
\left(\frac{d C_{A}}{d t}\right)_{t_{0}}=\frac{-3 C_{A 0}+4 C_{A 1}-C_{A 2}}{2 \Delta t}
$$

Pontos intermediários:

$$
\left(\frac{d C_{A}}{d t}\right)_{t_{i}}=\frac{4 C_{A(i+1)}-C_{A(i-1)}}{2 \Delta t}
$$

Ponto final:

$$
\left(\frac{d C_{A}}{d t}\right)_{t n}=\frac{C_{A n-2}-4 C_{A n-1}+3 C_{A n}}{2 \Delta t}
$$

A partir das equações (3) e (4) são calculadas as derivadas no primeiro ponto e nos pontos intermediários, respectivamente, enquanto a equação (5) é usada para calcular a derivada no ponto final (FOGLER, 2013). Finalmente, para a obtenção dos valores dos parâmetros cinéticos, basta plotar $\ln \left(-d C_{A} / d t\right)$ vs $\ln \left(C_{A}\right)$, no qual a ordem é obtida pela inclinação da reta e $k$ a partir da exponencial do coeficiente linear obtido.

\section{Método Integral}

Para a determinação dos parâmetros cinéticos de uma reação pelo método da integral, supõe-se uma ordem de reação na equação (1) e tal equação diferencial é integrada definidamente (PEREIRA, 2019). As equações (6)-(8) são as equações integradas supondo as ordens de reação 0,1 e $n \geq 2$, respectivamente, que estão dispostas no quadro 1 (FOGLER, 2013).

QUADRO 1- Equações utilizadas no Método da Integral para ordem 0, 1 e $n \geq 2$

\begin{tabular}{|ccc|}
\hline Ordem $(\boldsymbol{n})$ & Equação Integrada \\
\hline 0 & $C_{A}=C_{A 0}-k t$ & (6) \\
\hline 1 & $\ln \left(\frac{C_{A 0}}{C_{A}}\right)=k t$ \\
\hline
\end{tabular}




$$
n \geq 2 \quad \frac{1}{(n-1) C_{A}{ }^{n-1}}=\frac{1}{(n-1) C_{A_{0}}^{n-1}}+k t
$$

Se a suposta ordem de reação for correta, o gráfico correspondente dos dados de concentração versus tempo deve ser linear, ou seja, deve apresentar um coeficiente de regressão linear $\left(\mathrm{r}^{2}\right)$ o mais próximo de um possível. $O$ valor da constante cinética $(k)$ corresponde à inclinação da reta obtida (FOGLER, 2013).

\section{Método das Taxas Iniciais}

A velocidade inicial, $-r_{A 0}$, pode ser calculada por diferenciação dos dados e posterior extrapolação para o tempo zero. A lei da velocidade linearizada que relaciona $C_{A 0}$ com $-r_{A 0}$ é expressa pela equação (9) (FOGLER, 2013).

$$
\ln \left(-r_{A 0}\right)=\ln (k)+n \ln \left(C_{A 0}\right)
$$

A inclinação da reta do gráfico $\ln \left(-r_{A 0}\right)$ vs $\ln \left(C_{A 0}\right)$ fornecerá a ordem da reação $(n)$ e aplicando exponencial no coeficiente linear obtém-se o valor da constante de velocidade $(k)$.

\section{Método dos Mínimos Quadrados}

Esse método pode ser utilizado quando a taxa depende da concentração de mais de uma espécie. Considerando uma reação do tipo $A+B \rightarrow P$, a equação da taxa é dada pela equação (10) (FOGLER, 2013).

$$
-r_{A}=k c_{A}^{n} C_{B}^{n}
$$

em que $n$ é a ordem da reação em relação ao reagente $A, m$ é a ordem da reação em relação ao reagente $B$, e $C_{A}$ e $C_{B}$ são as concentrações molares dos reagentes $A$ e $\mathrm{B}$, respectivamente. A ordem global da reação é dada por $n+m$ (LEVENSPIEL, 2011). Linearizando a equação da taxa determina-se a equação (11).

$$
\ln \left(-\frac{d C_{A}}{d t}\right)=\ln (k)+n \ln \left(C_{A}\right)+m \ln \left(C_{B}\right)
$$

Ao resolver sistema gerado pelas equações (12)-(14) obtém-se os parâmetros $a_{0}, a_{1} e a_{2}$ que corresponde a $\ln (k), n$ e $m$, respectivamente.

$$
\begin{aligned}
& \sum_{j=1}^{n} Y_{j}=a_{0} N+a_{1} \sum_{j=1}^{n} x_{1 j}+a_{2} \sum_{j=1}^{n} x_{2 j} \\
& \sum_{j=1}^{n} x_{1 j} Y_{j}=a_{0} \sum_{j=1}^{n} x_{1 j}+a_{1} \sum_{j=1}^{n} x_{1 j}^{2}+a_{2} \sum_{j=1}^{n} x_{1 j} x_{2 j}
\end{aligned}
$$


$\sum_{j=1}^{n} x_{2 j} Y_{j}=a_{0} \sum_{j=1}^{n} x_{2 j}+a_{1} \sum_{j=1}^{n} x_{1 j} x_{2 j}+a_{2} \sum_{j=1}^{n} x_{2 j}^{2}$

no qual $Y=\ln \left(-\frac{d C_{A}}{d t}\right), N$ é o número de pontos, $x_{1}=\ln \left(C_{A}\right)$, e $x_{2}=\ln \left(C_{B}\right)$.

Vale ressaltar que, se a taxa de reação for dependente de apenas um termo de concentração o sistema se reduz às equações (12) e (13) apenas, sem os termos de dependência de $a_{2}$.

A determinação dos parâmetros cinéticos é de extrema importância para o estudo do comportamento de uma reação química, uma vez que a taxa de reação é função desses parâmetros. Para a determinação desses parâmetros, é fundamental dispor de dados coletados durante uma reação química e, dependendo da característica desses dados, o método para a determinação de tais parâmetros pode variar.

Para determinação dos coeficientes das curvas fornecidas por cada método apresentado, utilizou-se o método numérico de ajuste de curva proposto por Ruggiero (1996), no qual consiste em fazer a determinação dos parâmetros de uma curva através do método dos mínimos quadrados. Para a criação das janelas de acesso do usuário utilizou-se alguns recursos disponíveis no Scilab, além da utilização de outras funções já definidas no software.

O código foi criado de forma que o usuário possa selecionar o tipo de reação, o tipo de dado coletado no levantamento cinético, bem como o método para a determinação dos parâmetros cinéticos. Logo, é importante que o usuário se atente às características do problema de forma a escolher o melhor método para o cálculo de tais parâmetros.

\section{Estudos de Caso}

Para validar os códigos e, consequentemente, mostrar a eficiência dos mesmos, foi realizado alguns estudos de caso aplicado à cada método citado. Dessa forma, foi possível comprovar a veracidade dos resultados obtidos pelos códigos quando comparados com a literatura.

taxa simples

Estudo de Caso 1- Determinação dos Parâmetos Cinéticos para reações de

Tomando como base uma reação $A \rightarrow B$ realizada em fase líquida em um reator batelada no qual foi medido a variação da concentração de reagente limitante com o tempo. Tais dados são mostrados na Tabela 1 (Adaptado FOGLER, 2013).

TABELA 1- Levantamento cinético

\begin{tabular}{c|c|c|c|c|c|c|c}
\hline $\mathbf{T}(\mathbf{m i n})$ & 0 & 50 & 100 & 150 & 200 & 250 & 300 \\
\hline $\mathbf{C}_{\mathbf{A}}(\mathbf{m o l} / \mathbf{L})$ & 0,05 & 0,038 & 0,0306 & 0,0256 & 0,0222 & 0,0195 & 0,0174 \\
\hline
\end{tabular}

Este problema trata de reações com taxa simples, ou seja, a taxa é função da concentração de apenas um reagente. Os dados coletados no levantamento cinético correspondem à variação da concentração do reagente em função do tempo.

Estudo de Caso 2- Determinação dos Parâmetos Cinéticos para o levantamento cinético em função das taxas iniciais 
Utilizando a análise de mínimos quadrados e método das taxas iniciais, encontre os parâmetros cinéticos dos dados dispostos na Tabela 2 (Adaptado de SILVA, 2016).

TABELA 2- Variação da taxa inicial de reação em relação à concentração inicial de reagente limitante

\begin{tabular}{c|c|c|c|c|c}
\hline $\mathbf{C}_{\mathbf{A} \mathbf{0}}(\mathbf{m o l} / \mathbf{L})$ & 0,1 & 0,5 & 1 & 2 & 4 \\
\hline $\begin{array}{c}-\mathbf{r}_{\mathbf{A} 0} \\
\left(\mathbf{m o l} / \mathbf{h} \cdot \mathbf{m}^{2}\right) \mathbf{1 0}\end{array}$ & 0,073 & 0,7 & 1,84 & 4,86 & 12,84 \\
\hline
\end{tabular}

O levantamento cinético foi feito a partir da medição da taxa de reação nos instantes iniciais da reação, dessa forma, pode-se utilizar o método dos mínimos quadrados para reações com taxa simples ou o método das taxas iniciais para a determinação da ordem e da constante cinética da reação em questão.

Estudo de Caso 3- Determinação dos Parâmetos Cinéticos para reações químicas com taxa composta

A hidrogenólise do etano usando catalisador de níquel foi realizada 6 corridas com $\mathrm{H}_{2}$ e etano sendo alimentados no reator a $300^{\circ} \mathrm{C}$ (Tabela 3). Admitindo-se que a equação da taxa é $-r_{A}^{\prime}=K P_{A}^{\alpha} P_{B}^{\beta}$, determine os parâmetros da equação da taxa (Adaptado de SILVA, 2016).

TABELA 3- Variação da pressão de reagente em função da taxa

\begin{tabular}{c|c|c|c|c|c|c}
\hline $\mathbf{P}_{\mathbf{A}}$ (atm) & 0,475 & 0,465 & 0,420 & 0,320 & 0,54 & 0,570 \\
\hline $\mathbf{P}_{\mathbf{B}}$ (atm) & 0,475 & 0,465 & 0,420 & 0,520 & 0,540 & 0,370 \\
\hline$-\mathbf{r}_{\mathrm{A}}$ (mol/gcat.h) & 1,062 & 1,05 & 1,20 & 0,60 & 0,9375 & 0,0625 \\
\hline
\end{tabular}

A taxa da reação em questão é função da concentração de dois reagentes, $A$ e $B$, assim, o método dos mínimos quadrados deve ser utilizado para determinar a ordem da reação em relação à cada reagente, assim como a constante cinética.

\section{RESULTADOS E DISCUSSÃO}

Desenvolvimento do Site CRsolve

A fim de disponibilizar os códigos à, principalmente, alunos do curso de Engenharia Química, um site entitulado CRsolve foi criado contendo os códigos desenvolvidos neste trabalho. No site também pode ser encontrado o tutorial de como utilizar tais códigos, além de dar acesso ao download do software Scilab, visto que é imprescindível que esse software esteja instalado no computador do usuário, uma vez que todos os códigos foram desenvolvidos nessa plataforma. O link para acesso ao site é: <https://crsolve.wixsite.com/solve> e assim o usuário poderá fazer o download dos códigos em seu computador utilizá-los como ferramenta de estudo. 
Para ter acesso aos códigos, primeriamente o usuário deve fazer o download através do site CRSOLVE que apresenta uma interface simples e de fácil acesso. Foi criada a versão desktop e versão mobile do site, porém, por questões de compatibilidade, somente a versão desktop apresenta as opções de download dos códigos. A figura 1 mostra a tela inicial do site CRSOLVE.

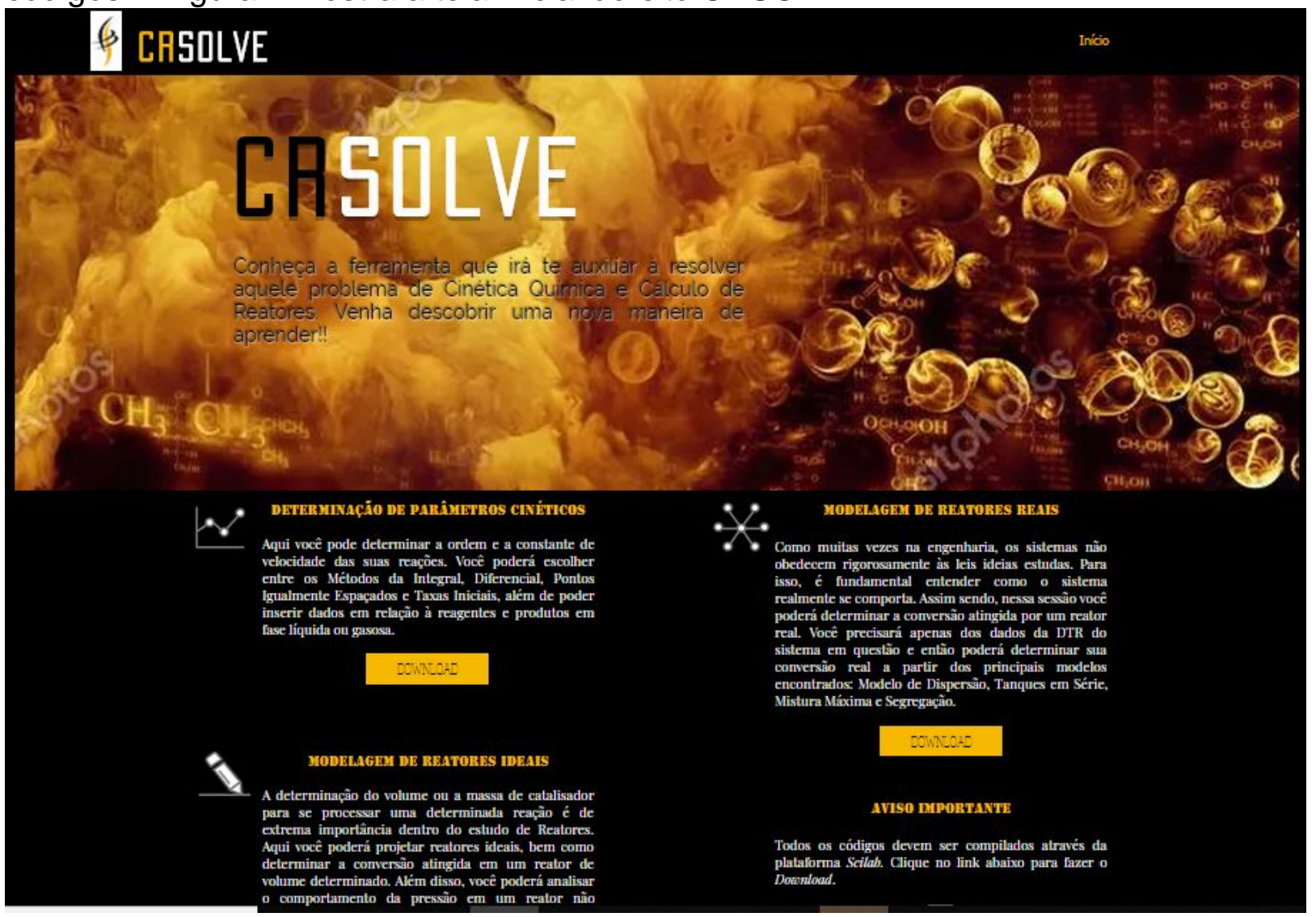

FIGURA 1- Interface do site CRSOLVE versão desktop.

Como visto na figura 1, no site encontram-se outros códigos para a solução de outros problemas relacionado ao projeto e análise de desempenho de reatores reais e ideais não abordado nesse trabalho. Além das opções de download dos códigos, há um link para efetuar o download do software livre Scilab e uma seção para contato com o desenvolvedor.

Feito o download dos códigos, e já com o sofwtare Scilab instalado no computador do usuário, é possível compilar o arquivo e, de acordo com o problema a ser resolvido, selecionar o melhor método de determinação dos parâmetros cíneticos. Os resultados obtidos após compilar os códigos de cada seção estão dispostos a seguir. Assim, foi possível analisar e comparar os resultados obtidos pelos códigos, para cada estudo de caso, com aqueles encontrados na literatura.

\section{Estudo de Caso 1}

Ao compilar o código uma mensagem de aviso aparecerá, informando o usuário que as variáveis podem ser inseridas em qualquer unidade desde que elas sejam compatíveis. Após aceitar tal condição é fundamental especificar qual o tipo de dado coletado no levantamento cinético, no qual pode ser em função dos reagentes, produtos ou pressão total do sistema. Antes de inserir os dados, é 
necessário escolher o método que se deseja calcular as constantes cinéticas como é mostrado na figura 2.

Mensagem do Scilab

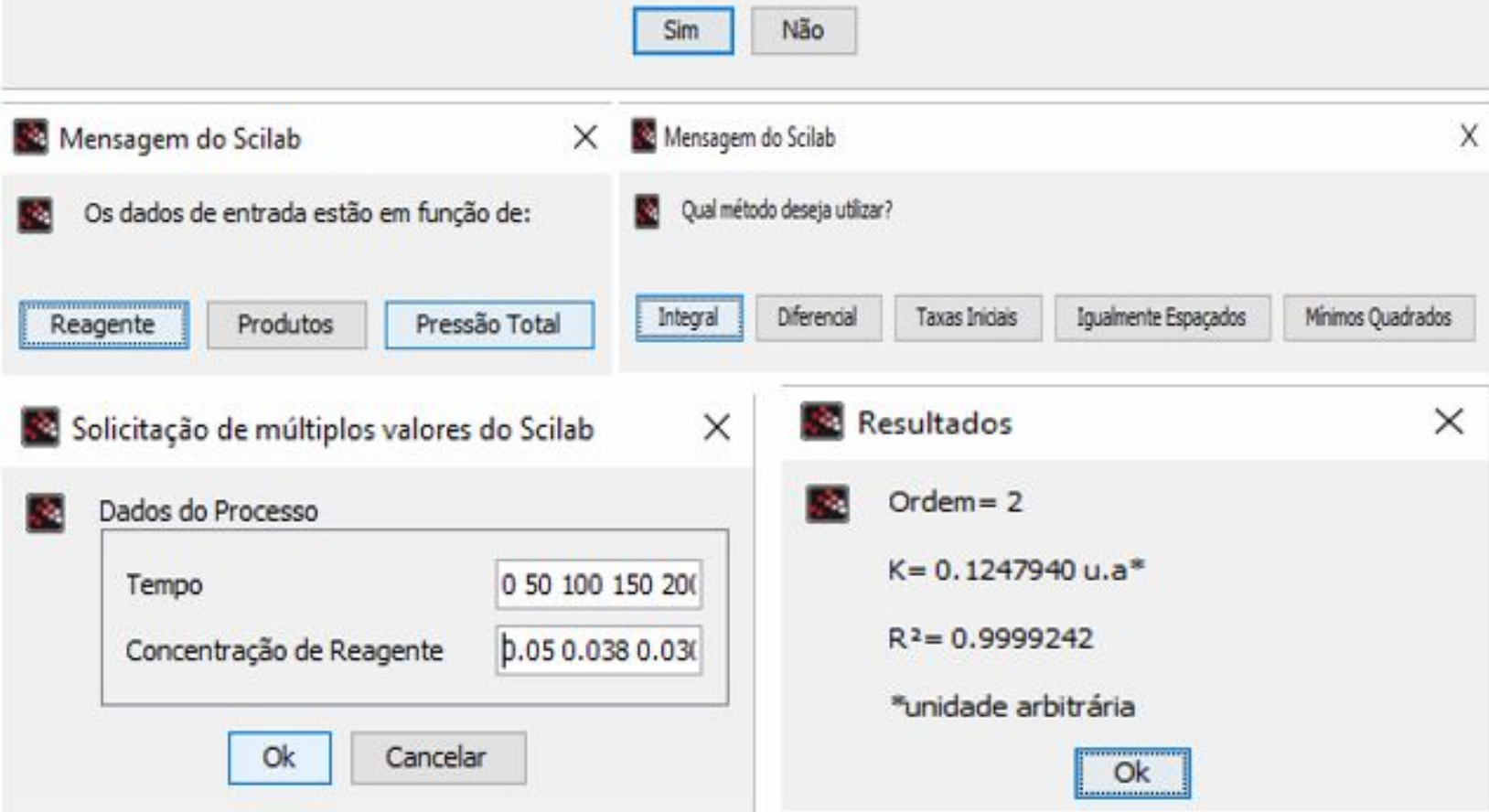

FIGURA 2- Janelas informativas de entrada de dados e resultados do problema.

A Tabela 4 compara o resultado obtido através da resolução encontrado na literatura e os resultados proveniente dos códigos, utilizando os Métodos Integral, Diferencial e Pontos Igualmente Espaçados. Vale ressaltar que, como o usuário poderá entrar com os dados em qualquer unidade, a unidade da constante cinética será correspondente àquelas inseridas.

TABELA 4- Comparativo entre os Métodos Integral, Diferencial e Pontos Igualmente Espaçados

\begin{tabular}{c|c|c|c|c}
\cline { 2 - 4 } & $\begin{array}{c}\text { Ordem } \\
(\mathbf{n})\end{array}$ & $\begin{array}{c}\text { Constante } \\
\mathbf{d e} \\
\text { Velocidade } \\
\mathbf{( k )}\end{array}$ & $\begin{array}{c}\text { Coeficiente de } \\
\text { Regressão (r) }\end{array}$ & $\begin{array}{c}\text { Erro } \\
\text { Absoluto (\%) } \\
\text { referente ao k } \\
\text { encontrado } \\
\text { na literatura }\end{array}$ \\
\hline $\begin{array}{c}\text { Integral } \\
\text { (FoGLER, 2013) }\end{array}$ & 2 & 0,1248 & 0,9993 & - \\
\hline $\begin{array}{c}\text { Integral (códigos } \\
\text { do autor) }\end{array}$ & 2 & 0,1247 & 0,9998 & 0,01 \\
\hline $\begin{array}{c}\text { Diferencial } \\
\text { (códigos do } \\
\text { autor) }\end{array}$ & 2,02 & 0,1331 & 0,9990 & 0,83 \\
\hline $\begin{array}{c}\text { Pontos } \\
\text { Igualmente } \\
\text { Espaçados }\end{array}$ & 1,995 & 0,1234 & 0,9942 & 0,14 \\
\hline
\end{tabular}




\begin{tabular}{c|l|l|l|l}
\hline $\begin{array}{c}\text { (códigos do } \\
\text { autor) }\end{array}$ & & & & \\
\hline
\end{tabular}

Estudo de Caso 2

A entrada de dados é de forma análoga ao Estudo de Caso 1, variando apenas - Método de resolução, que nesse caso pode ser pelo Método dos Mínimos Quadrados ou Método das Taxas Iniciais. A tabela 5 mostra o comparativo usando o método de Mínimos Quadrados e das Taxas Iniciais para determinar parâmetros cinéticos de uma reação com taxa simples referente ao Estudo de Caso 2.

TABELA 5- Comparativo entre resultados utilizando o Método dos Mínimos Quadrados e das Taxa Iniciais

\begin{tabular}{c|c|c|c|c}
\cline { 2 - 4 } & $\begin{array}{c}\text { Ordem } \\
(\mathbf{n})\end{array}$ & $\begin{array}{c}\text { Constante de } \\
\text { Velocidade }(\mathbf{k})\end{array}$ & $\begin{array}{c}\text { Coeficiente } \\
\text { de Regressão } \\
\left(\mathbf{r}^{2}\right)\end{array}$ & $\begin{array}{c}\text { Erro Absoluto } \\
\mathbf{\%} \% \text { referente } \\
\text { ao k } \\
\text { encontrado na } \\
\text { literatura }\end{array}$ \\
\hline $\begin{array}{c}\text { Mínimos } \\
\text { Quadrados } \\
\text { (literatura) }\end{array}$ & 1,4 & 0,0184 & - & - \\
\hline $\begin{array}{c}\text { Mínimos } \\
\text { Quadrados } \\
\text { (códigos do } \\
\text { autor) }\end{array}$ & 1,401 & 0,01841 & - & 0,001 \\
\hline $\begin{array}{c}\text { Taxas Iniciais } \\
\text { (códigos do } \\
\text { autor) }\end{array}$ & 1,4 & 0,01841 & 0,9999 & 0,001 \\
\hline
\end{tabular}

Estudo de Caso 3

Analogamente aos Estudo de Caso 1 e 2, ocorre a entrada de dados no Estudo de Caso 3, porém, como a taxa da reação é composta é necessário usar o Método dos Mínimos Quadrados para a determinação dos parâmetros cinéticos dessa reação. A Tabela 6 mostra os resultados para uma reação com taxa composta referente ao Estudo de Caso 3.

TABELA 6- Comparativo entre resultados utilizando o Método dos Mínimos Quadrados para reações químicas com taxa composta

\begin{tabular}{c|c|c|c|c|c}
\cline { 2 - 5 } & $\begin{array}{c}\text { Ordem } \\
(\boldsymbol{\alpha})\end{array}$ & $\begin{array}{c}\text { Ordem } \\
(\boldsymbol{\beta})\end{array}$ & $\begin{array}{c}\text { Ordem } \\
\text { Global }\end{array}$ & $\begin{array}{c}\text { Constante } \\
\mathbf{d e} \\
\text { Velocidade } \\
\mathbf{( k )}\end{array}$ & $\begin{array}{c}\text { Erro Absoluto } \\
(\%) \text { referente } \\
\text { ao k } \\
\text { encontrado } \\
\text { na literatura }\end{array}$ \\
\hline $\begin{array}{c}\text { Mínimos } \\
\text { Quadrados } \\
\text { (literatura) }\end{array}$ & 0,9525 & 2,008 & 2,9605 & 0,4793 & - \\
\hline $\begin{array}{c}\text { Mínimos } \\
\text { Quadrados } \\
\text { (códigos do } \\
\text { autor) }\end{array}$ & 0,984 & 1,957 & 2,941 & 0,5112 & 3,19 \\
\hline
\end{tabular}




\section{Discussão}

Quando se trata de uma reação cuja taxa é função da concentração de apenas um reagente a ordem global da reação corresponde à ordem em relação à esse reagente, porém, quando a taxa é composta, ou seja, é função da concentração de dois ou mais reagentes a ordem global da reação é a soma das ordens referente à cada reagente, como pode ser visto na Tabela 7.

Analisando os resultados mostrados nas Tabelas 4, 5 e 6 observa-se que a diferença entre os resultados da literatura e os calculados pelos códigos é pequena. Tal diferença pode ser justificada pelo fato de que o número de casas decimais utilizada pelo código é muito maior que aquele utilizado nas soluções da literatura. Logo, a implementação dos códigos em sala de aula torna-se uma ferramenta de auxílio na correção de exercícios ou até mesmo no desenvolvimento de projetos dessa área.

Dentre os métodos analisados, o método de mínimos quadrados para taxa composta apresentou o maior erro absoluto, uma vez que a resolução de tal método leva em consideração a variação de duas concentrações em função da variação da taxa de reação.

\section{CONCLUSÃO}

O desenvolvimento de códigos com característica de acesso indireto do usuário requer cautela, uma vez que é fundamental prever qualquer tipo de erros que podem ser gerados além de qualquer variação do processo estudado. Todavia, dentro das limitações apresentadas, os códigos forneceram bons resultados quando comparados aos encontrados na literatura, apresentando um erro absoluto médio em relação ao $k$ menor que $1 \%$, que pode ser explicado pela discrepância entre 0 número de casas decimais utilizado pelo código em cada método em relação ao número de casas decimais utlizados na literatura.

O resultado que aprentou maior erro absoluto foi o método dos Mínimos Quadrados para reações com taxa composta, com um valor de 3,19\%. Esse erro pode ser justificado pelo fato do método levar em consideração a influência da variação da concentração de dois reagentes, além da necessidade da resolução de um sistema de equações $3 \times 3$ que, mais uma vez, é influenciado pelo número de casas decimais utilizado pelo código.

Ademais, as técnicas de resolução de problemas através de plataformas computacionais são de extrema importância em todas as áreas da Engenharia, uma vez que a utilização dessas técnicas promovem, não só a sedimentação da teoria abordada em sala de aula, como também o desenvolvimento do raciocínio lógico dos alunos.

Portanto, a utilização de recursos computacionais para a resolução de problemas de Engenharia dever ser cada vez mais difundida, uma vez que a aliança entre tais recursos e a teoria abordada em sala de aula é capaz de dispertar o senso crítico dos alunos e assim a formação de engenheiros capazes de tomar decisões plausíveis diante à uma adversidade.

A fim de avaliar os códigos desenvolvidos, vê-se a necessidadeda aplicação dos mesmos em sala de aula como ferramenta de estudo. Assim, os discentes e 
docentes poderão avaliar e sugerir melhorias para que os códigos tornem-se cada vez mais didáticos e eficientes.

\section{REFERÊNCIAS}

ANGRISANO, L.C. O papel do engenheiro químico na sociedade. Revista Dom Total, 2016. Dispopnível em:< http://domtotal.com/noticia/1095590/2016/11/o-papeldo-engenheiro-quimico-na-sociedade/>. Acessado em: 29/07/2019.

FERREIRA, I. E. P.; TRINCA, L. A.; FERREIRA, C. P.. Delineamentos experimentais eficientes para estudos de cinética química. Scielo-Quím. Nova vol.37, nำ- São Paulo, 2014. Disponível em:< http://quimicanova.sbq.org.br/imagebank/pdf/v37n4a03.pdf>. dx.doi.org/10.5935/0100-4042.20140102.

FOGLER, H. S. Elementos da engenharia das reações químicas. 4.ed. Rio de Janeiro: LTC, 2013.

LEVENSPIEL, O. Engenharia das reações químicas. São Paulo: Bluncher, 2011.

MAYNARD, A. D. Navigating the fourth industrial revolution. Nature nanotechnology, v. 10, n. 12, p. 1005, 2015. Disponível em:< https://www.nature.com/articles/nnano.2015.286>. DOI: 10.1038/nnano.2015.286.

PEREIRA, M.A. Cinética Química: Introdução à Cinética. Escola de Engenharia de Lorena- USP. $\quad$ Capítulo $1 . \quad$ Disponível em:< http://www.marco.eng.br/cinetica/notasdeaula/capitulo-1.pdf>. Acessado em: 23/07/2019.

ROSEN, R.; VON WICHERT, G.; LO, G.; BETTENHAUSEN, K. D. About the importance of autonomy and digital twins for the future of manufacturing. IFACPapersOnLine, v. 48, n. 3, p. 567-572, 2015. Disponível em: < https://doi.org/10.1016/j.ifacol.2015.06.141>. DOI: 10.1016/j.ifacol.2015.06.141.

RUGGIERO, M.A. G.;LOPES, V. L. R.. Cálculo numérico: aspectos teóricos e computacionais. $2^{\underline{a}}$ ed. Makron. 1996.

RÜBMANN, M.; LORENZ, M.; GERBERT, P.; WALDNER, M.; JUSTUS, J.; ENGEL, P.; HARNISCH, M. Industry 4.0: The future of productivity and growth in manufacturing industries. Boston Consulting Group, v. 9, 2015.

SAKURAI, R.; ZUCHI, J. D. As Revoluções Industriais até a Indústria 4.0. Revista Interface Tecnológica, v. 15, n. 2, p. 480-491, 30 dez. 2018. Disponível em:< https://revista.fatectq.edu.br/index.php/interfacetecnologica/article/view/386>. DOI: 10.31510/infa.v15i2.386.

SCHMAL, M. Cinética e reatores: aplicação na engenharia química: teoria e exercícios. 2.ed.- Rio de Janeiro: Synergia: COPPE/UFRJ, 2013. 
SCHULTZ, G.; LAUER, J.; LOPES, J. M.; RANGEL, R. S.; SIMONELLI, G. Modelagem e Simulação dos Reatores Químicos BR e PFR no EMSO. Enciclopédia Biosfera, Centro Científico Conhecer - Goiânia, v.10, n.18; p. 3756. 2014.

http://www.conhecer.org.br/enciclop/2014a/ENGENHARIAS/MODELAGEM.pdf>.

SCILAB. What is Scilab. Available in < http://scilab.io/products/scilab/>. Acessado em: $23 / 07 / 2019$.

SILVA, P.P. Cinética Química e Cálculo de Reatores II. Notas de Aula. Universidade Federal do Triângulo Mineiro- ICTE. Uberaba, 2016.

SILVEIRA; B. I.. Cinética química das reações homogêneas. 2. Ed. Revista e ampliada- São Paulo: Bluncher, 2015.

SOUZA, T. V. F.; RODRIGUES, B. P.; BRITO, I. A. M.; NASCIMENTO, L.; MELNYK, A.. A reatividade da cinética química na reação do ácido clorídrico com alumínio. Revista Mangaio Acadêmico, v. 1, n. 2, jul/dez, 2016. Disponível em:< http://revistaadmmade.estacio.br/index.php/mangaio/article/viewFile/3066/1391>. 